Review Article

\title{
Mathematical Studying in Control of Grasping
}

\author{
Eman Mirzaieepoor ${ }^{1}$, Mohammad Sadegh Hassanli ${ }^{2}$, Pezhman Moradi ${ }^{3}$, \\ Mohammad Mehdi Khoddami ${ }^{3}$ \\ ${ }^{1}$ Department of Mechanical Engineering, Amirkabir University, Tehran, Iran \\ ${ }^{2}$ Department of Exercise Physiology, Jahrom University, Jahrom, Iran \\ ${ }^{3}$ Department of Mechanical Engineering, Jahrom University, Jahrom, Iran
}

Email address:

Emirzaieepoor@gmail.com (E. Mirzaieepoor),Pezhman_moradi123@yahoo.com (P. Moradi)

To cite this article:

Eman Mirzaieepoor, Mohammad Sadegh Hassanli, Pezhman Moradi, Mohammad Mehdi Khoddami. Mathematical Studying in Control of Grasping. International Journal of Science and Qualitative Analysis. Vol. 2, No. 1, 2016, pp. 1-13. doi: 10.11648/j.ijsqa.20160201.11

Received: March 15, 2016; Accepted: June 3, 2016; Published: July 5, 2016

\begin{abstract}
The main purpose of developing of mechanical hands is to give robots the knack in order to grasp objects of varying geometric and physical properties. The complete model is a coupling of models which describe contact behavior with generally using the models of rigid-body kinematics and dynamics. The contact model fundamentally come down to the choice of components of contact force and moment which are transmitted through each contact. Mathematical properties of the complete model obviously bring about two primary grasp types whose physical interpretations provide insight for grasping and manipulation planning. A grasp with complete restraint avoids loss of contact and therefore is so secure. As will be mentioned, two primary limitation properties are force closure and form closure. A form closure grasp assurances the maintenance of contact as long as the links of the hand and the proposed object are also well verged on as rigid and as long as the joint actuators are sturdy enough. It should be noted that the main difference between force closure and also form closure grasps is the latter's reliance on contact friction.
\end{abstract}

Keywords: Computational Linear algebra, Linear Kinematic, Linear Dynamic, Grasping, Rigid-body Models, Form Closure

\section{Introduction}

A grasp is commonly defined as a set of various contacts models on the surface of the object, which purpose is to constrain the potential motions of the object in the event of external disorders [1-4].Grasping is a typical human capability that is practical in robotic hand system nowadays and is employed in a variety of ways. Except the human hands many biological model exists in nature that they can be used in different sectors. A sample of the biomimetic systems from the motion mechanism of animals are used in biomimetic micro-robots [5-9]. In particular, the three most important functions are to restrain objects, to explore and to manipulate objects. For grasping motion control of robotic two abilities, hands tactile and slippage sensations are very important and difficult [10]. To successfully manipulate objects in carry out many complex manipulation tasks, robot systems require some form of tactile feedback to distinguish the object's hardness, structure and geometrical position [11]. For example, some items may be soft and light, for example a stuffed animal or a void cardboard box, while others might be dense and hard, such as a glass bottle or testing tool set (knives) as presented in Figure. 1 [12].

For a specific robotic hand, different grasp types are planned and analyzed in order to decide which one to execute. Given these 2D points in each image, we use triangulation to obtain a 3D position at which to actually attempt the grasp.

Figure 2 displays the examples of grasping found for various objects [13]. Thus, rather than trying to triangulate every single point within each image in order to estimate the depths. We merely try to triangulate one point corresponding to the 3D point where we will grasp the desire object [14].

If we want to grasp a formerly known object, or if we can attain a full 3D model of the object, next several approaches for instance ones based on friction cones $[15,16]$, form- and force-closure [17-21], pre-stored primitives [22], or other methods can be applied, and the rest of paper is organized as follows:

Section 2 states the grasp analysis based on Form closure. 
In order to investigate it carefully, we divided this section into four parts and mathematical studying are conducted in detail.

Section 3 establishes grasp analysis based on Force closure. A grasp has force closure, or is force closed, if the grasp can be maintained in the face of any object wrench. Force closure is the same as the form closure, however relaxed to permit friction forces to help balance the object wrench. A benefit of including friction in the analysis is the reduction in the number of contact points needed for closure As will be seen, this segment divided into three parts, and like previous section, mathematical studying are done.

Section 4 proposes "Grasped Polygon in the Plane". As can be expected grasping of polygon is difficult at the moment. In order to achieve our purpose, simple mathematical studying based on the proposed methods are introduced. Later "Hyperstatic Grasps" is presented in depth finally, concluding remarks are presented.
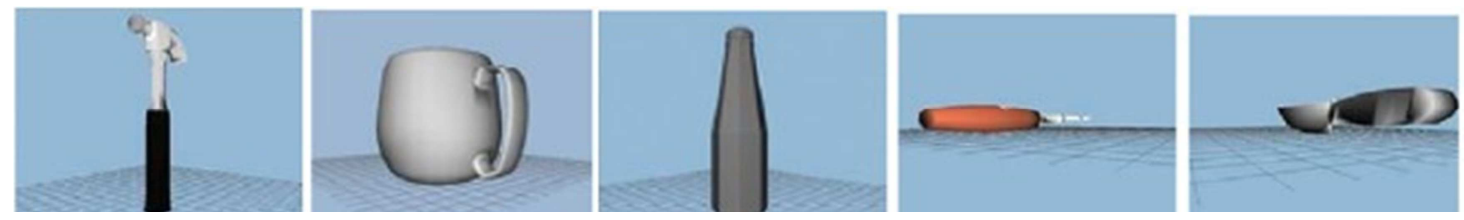

Fig. 1. Examples of unstable grasps from best single approach.
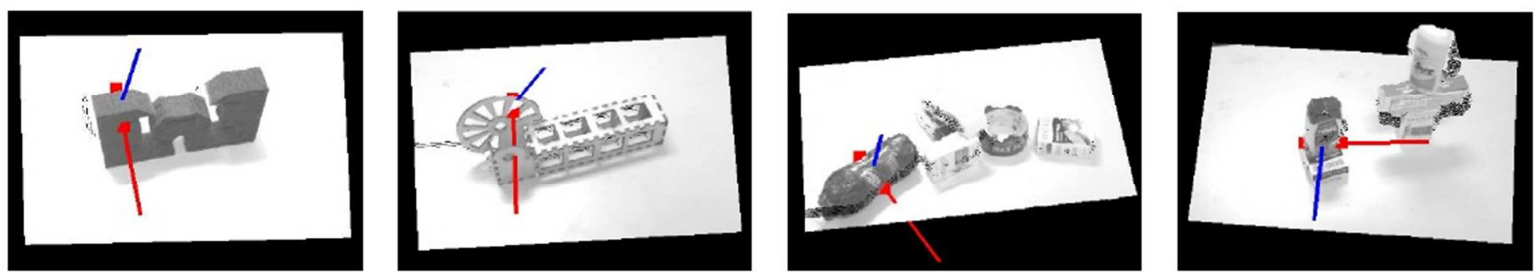

Fig. 2. 3D visualizations of the best detected grasp in several scenes. The colored axes represent the orientation of the grasp (blue is along the gripper wrist and red is normal to the gripper pads (as shown in Figure 3).

\section{Human Hand Behaviour}

\subsection{Grasping Stability of an Object}

Concluded that the detection of the absence, incipence, or occurrence of slip are essential for human beings to grasp and lift a fragile object. By experiments in humans and chimpanzees, they showed that when the finger presses an object or when the object starts moving before slipping [23], the skin stretches and the rapidly adapting (RAs), slowly adapting (SAs) and paining corpuscle (PCs) receptors are activated. The RAs and PCs respond only in the initial skin stretching phase (absence or incipience of slip) [24] and are inactive when the relative velocity of the object is not zero (when the object slips). Only the SAs receptors continue the respond in the slip situation.In addition, it is shown that the receptor response depends on the velocity of the relative motion between the object and the finger. So when a subject lifts an object, at first the skin is stretched and then small distinct slips that vibrate the object are observed. Each of those slips are strong enough to activate the mechanoreceptors and cause an automatic increase in the gripping force, in order to prevent the continuation of the relative motion of the object with respect to the skin. Finally, a human being perceives slip and incipient slip situation and operates its fingers to grasp stably an object.

\subsection{Lift and Hold}

After stable contact is achieved, the controller transitions to the next grasp phase: Lift and Hold. In this phase, the robot holds the object between its fingertips and moves its arm to accomplish higher level tasks. It is desirable for the grasp controller to hold the object firmly enough to avoid slipping, but gently enough to avoid crushing. As with FA-I signals in human grasping, the high-pass filtered force signal $\widetilde{F}_{g}$ is a strong indicator of slip. This signal is more reliable than $F_{g}$ itself, since it does not vary significantly with low-frequency robot motion and reorientation of the object with respect to gravity. We calculate the Slip condition as follows:

$$
\text { Slip } \left.=\left(\left|\widetilde{F}_{g}\right|>F_{g} \cdot \text { SLIPTHRESH }\right) \& \& F_{g}^{B P}<\text { FBPTHRESH }\right)
$$

Our Slip condition is met only when both subsidiary sensory comparisons evaluate to true. First, the magnitude of the force disturbance signal $\tilde{F}_{g}$ must exceed a threshold that is defined by the product of the total force $F_{g}$ and a constant SLIPTHRESH; using this product rather than a constant value makes the robot less sensitive to force variations as the grip force increases.

This approach was again inspired by human capabilities: Human force perception is known to follow Weber's law, where a stimulus must vary by a certain percent of its magnitude to have the change be detectable [25].

Second, slips are considered only when the average grasp force does not change very quickly. This force stability condition was evaluated by treating the average finger pad force $F_{g}$ with a first-order Chebyshev discrete-time band-pass filter with a pass band from 1 to $5 \mathrm{~Hz}$ to produce $F_{g}^{B P}$. This signal frequency cutoff of $1 \mathrm{~Hz}$ removes the mean 
value from the grip force signal, while the higher cutoff of 5 $\mathrm{Hz}$ removes the slip effects that are seen in $\tilde{F}_{g}$. This condition prevents the formation of a feedback loop when the controller increases its grip force to stop slip events, as discussed next. Every time Slip occurs, the controller increases the desired grip force $F_{c}$ by a small percentage of its current value, such that:

$$
F_{c}=F_{c} \cdot \mathrm{KSLIP}
$$

Several alternative methods to respond to slip were tested, such as increasing the desired grip force proportional to the magnitude of the slip event, as done by Takahashi et al. [26]. However, this signal depends somewhat on the properties of the grasped object; therefore, we use it in order to detect only that the grasp has been disturbed. In contrast with [26], we found that allowing the grip force to decay over time resulted in a higher percentage of dropped objects. This difference is most likely because of the slower response time of the PR2 gripper when compared with the hardware developed in [26]. This approach does cause the desire objects to be held tighter than the minimum required grasp force when transient slip conditions are experienced.

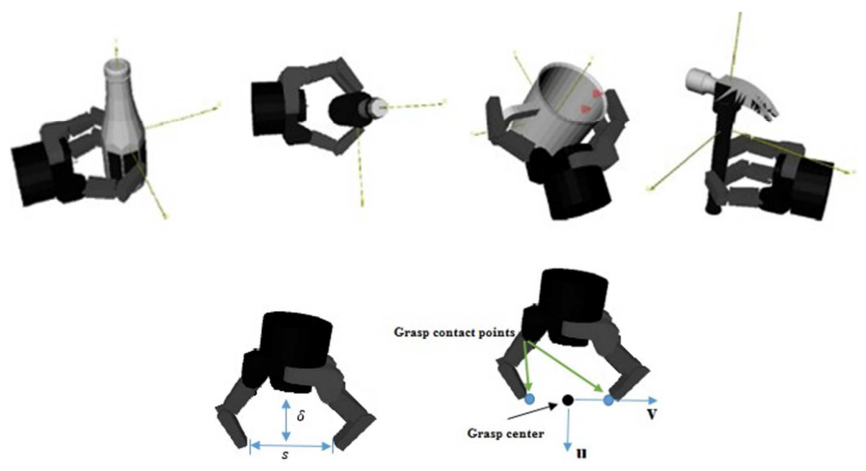

\section{Restraint Analysis}

The most vital necessities in grasping and dexterous manipulation $[27,28]$ are the abilities to hold an object firmly in equilibrium and control the position and orientation of the grasped object in regard to the palm of the hand as can be seen in Figure 4 [29]. The two most valuable characterizations of grasp limitation are force closure and form closure [30]. These terms (force closure and form closure) were used over 125 years ago in order to distinguish between joints which required an external force so as to maintain contact, and those that did not [31, 32]. For example, some water wheels had a cylindrical axle that was laid in a horizontal semi-cylindrical groove split on either side of the wheel. During operation, the weight of the wheel acted to close the groove-axle contacts, hence the term force closure. By contrast, if the grooves were replaced by cylindrical holes just long enough to accept the axle, then the contacts would be closed by the geometry(even if the direction of the gravitational force were reversed), hence the term form closure. When applied to grasping, form and force closure have the following interpretations. Assume that a hand grasping an object has its joint angles locked and its palm fixed in space; then the grasp has form closure, or the object is form closed, if it is impossible to move the object, even infinitesimally. Under the same conditions, the grasp has force closure, or the object is force closed, if for any noncontact wrench experienced by the object, contact wrench intensities exist that satisfy (20) and are consistent with the constraints imposed by the friction models applicable at the contact points. Notice that all form closure grasps are also force closure grasps. When under form closure, the object cannot move at all, regardless of the noncontact wrench [33]. Therefore, the hand maintains the object in equilibrium for any external wrench, which is the force closure requirement.

Fig. 3. Task-specific generated model grasps.
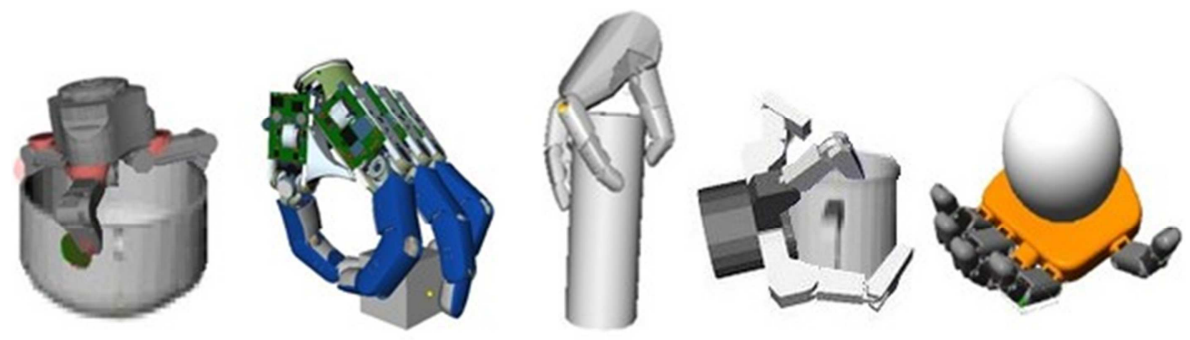

Fig. 4. Equilibrium and control the position and orientation of the grasped object relative to the palm of the hand.

Roughly speaking, form closure occurs when the palm and fingers wrap around the object forming a cage with no wiggle room such as the grasp shown in Fig. 5. This kind of grasp is also called a power grasp [34] or an enveloping grasp [35]. However, force closure is possible with fewer contacts, as shown in Fig. 4, but in this case force closure requires the ability to control internal forces. It is also possible for a grasp to have partial form closure, indicating that only a subset of the possible degrees of freedom are restrained by form closure [36]. An example of such a grasp is shown in Fig.8. In this grasp, fingertip placement between the ridges around the periphery of the gasoline cap provide form closure against relative rotation about the axis of the helix of the threads and also against translation perpendicular to that axis, but the other three degrees of freedom are restrained through force closure. Strictly speaking, given a grasp of a real object by a human hand, it is impossible to prevent relative motion of the object with respect to the palm due to the compliance of the hand and object. Preventing all motion is possible only if the contacting bodies are rigid, as is assumed in most mathematical models employed in grasp analysis. 


\subsection{Grasp Analysis}

The contact model can be used so as to investigate tasks involving multiple contacts. The set of contacts describing each grasp can be analyzed to test the grasp's ability. As will be mentioned later, the grasps can be maintained for every likely disturbing load are recognized as closure grasps. Note that this review paper only concentrate on the grasp analysis for one object to estimate the dexterity of the selected grasps and decide which one is the best to be performed.

\subsection{Form Closure}

To make the notion of form closure precise, introduce a gap function denoted by $\psi_{i}(u, q)$ at each of the $n_{c}$ contact points between the object and the hand. The gap function is zero at each contact, becomes positive if contact breaks, and negative if penetration occurs. The gap function can be thought of as the distance between the contact points. In general, this function is dependent on the shapes of the contacting bodies. Let $\bar{u}$ and $\bar{q}$ represent the configurations of the object and hand for a given grasp; then

$$
\psi_{i}(\bar{u}, \bar{q})=0 \forall i=1, \ldots, n \mathrm{c} .
$$

The form closure condition can now be stated in terms of a differential change $\mathrm{d} u$ of $\bar{u}$ :

A grasp (.u,.q) has form closure if and only if the following implication holds:

$$
\psi(\bar{u}+d u, \bar{q}) \geq 0 \Rightarrow d u=0,
$$

where $\psi$ is the $n_{c}$-dimensional vector of gap functions with $i-t h$ component equal to $\psi_{i}(u, q)$. By definition, inequalities between vectors imply that the inequality is applied between corresponding components of the Expanding the gap function vector in a Taylor series about. $u$ yields infinitesimal form closure tests of various orders.

Let $\beta \psi(u+q), \beta=1,2,3 \ldots$ denote the Taylor series approximation truncated after the terms of order $\beta$ in du. From (3), it follows that the first-order approximation is:

$$
1_{\psi}(\bar{u}+\mathrm{d} u, \bar{q})=\frac{\partial \psi(u, q)}{\partial u} \mid(\bar{u}, \bar{q})^{\mathrm{d} u},
$$

where $\partial \psi(u, q) / \partial u^{l}(\bar{u}, \bar{q})$ denotes the partial derivative of $\psi$ with respect to $u$ evaluated at $(\bar{u}, \bar{q})$.

Replacing $\psi$ with its approximation of order $\beta$ in (4) implies three relevant cases of order $\beta$ :

1. If there exists du such that $\beta \psi\left(u^{-}+d u, q^{-}\right)$has at least one strictly positive component, then the grasp does not have form closure of order $\beta$;

2. If for every nonzero du, $\beta \psi(\bar{u}+d u, \bar{q})$ has at least one strictly negative component, then the grasp has form closure of order $\beta$;

3. If neither case 1 nor case 2 applies for all $\alpha \psi(\bar{u}+$ $d u, \bar{q})) \forall \forall \alpha \leq \beta$, then higher-order analysis is required to determine the existence of form closure.

Figure. 8 illustrates form closure concepts using several planar grasps of gray objects by fingers shown as dark disks. The concepts are identical for grasps of three-dimensional objects, but are more clearly illustrated in a plane. The grasp on the left has first-order form closure. Note that first-order form closure only involves the first derivatives of the distance functions. This implies that the only relevant geometry in first-order form closure are the locations of the contacts and the directions of the contact normal. The grasp in the center has form closure of higher order, with the specific order depending on the degrees of the curves defining the surfaces of the object and fingers in the neighborhoods of the contacts [37]. Second-order form closure analysis depends on the curvatures of the two contacting bodies in addition to the geometric information used to analyze first-order form closure. The grasp on the right does not have form closure of any order, because the object can translate horizontally and rotate about its center.

\subsection{First-Order Form Closure [38]}

First-order form closure exists if and only if the following implication holds:

$$
\frac{\partial \psi(u, q)}{\partial u} \mid(\bar{u}, \bar{q})^{\mathrm{d} u \geq 0 \Rightarrow \mathrm{d} u=0 .}
$$

The first-order form closure condition can be written in terms of the object twist $v$ :

$$
G_{n}^{\top} v \geq 0 \Rightarrow v=0
$$

where $G_{n}^{\top}=\partial \psi / \partial u V \in R^{n c \times 6}$. because the gap functions only quantify distances, the product $\mathrm{G}$ is the vector of normal components of the instantaneous velocities of the object at the contact points (which must be nonnegative to prevent interpenetration). This in turn implies that the grasp matrix is the one that would result from the assumption that all contacts are of the type PwoF.

An equivalent condition in terms of the contact wrench intensity vector $\lambda_{n} \in R_{n c}$ can be stated as follows.

A grasp has first-order form closure if and only If:

$$
\left.\begin{array}{c}
G_{n} \lambda_{n}=-g \\
\lambda_{n} \geq 0
\end{array}\right\} \forall g \in \mathbb{R}^{6}
$$

The physical interpretation of this condition is that equilibrium can be maintained under the assumption that the contacts are frictionless. Note that the components of $\lambda_{n}$ are the magnitudes of the normal components of the contact forces. The subscript $(.)_{n}$ is used to emphasize that $\lambda_{n}$ contains no other force or moment components. Since $g$ must be in the range of $G_{n}$ for equilibrium to be satisfied, and since $g$ is an arbitrary element of R6, then in order for condition (8) to be satisfied, the rank of $G_{n}$ must be six. Assuming rank $\left(G_{n}\right)$ $=6$, another equivalent mathematical statement of first-order form closure is: there exists $\lambda_{n}$ such that the following two conditions hold [39]: 


$$
G_{n} \lambda_{n}=0, \lambda_{n}>0 .
$$

As can be inferred that there exists a set of firmly compressive normal contact forces in the null space of $G_{\mathrm{n}}$. In other words, one can press the object as tightly as desired while maintaining equilibrium. A second interpretation of this condition is that the nonnegative length of the columns of $G_{n}$ must equal $\mathrm{R}^{6}$.The duality of conditions (7) and (8) can be understood obviously by examining the set of wrenches that can be applied by frictionless contacts and the corresponding set of possible object twists [40].

\subsection{First-Order form Closure Requirements}

Several useful necessary conditions for form closure are known. In 1897 Somov proved that at least seven contacts are necessary to form close a rigid object with six degrees of freedom [41]. Lakshminarayana generalized this to prove that $n_{v}+1$ contacts are necessary to form close an object with $n_{v}$ degrees of freedom [41] (based on Goldman and Tucker 1956 [40]), see Table 1.

Table 1. Minimum number of contacts $n_{c}$ required to form close an object with $n_{v}$ degrees of freedom.

\begin{tabular}{lll}
\hline$n_{v}$ [42] & $n_{c}$ & {$[46]$} \\
3 (planar grasp) [43] & 4 & {$[47]$} \\
6 (spatial grasp) [44] & 7 & {$[48]$} \\
$n_{v}$ (general) [45] & $n_{v}+1[49]$ \\
\hline
\end{tabular}

Table.1 led to the definition of partial form closure that was mentioned above in the discussion of the hand grasping the gasoline cap. Markenscoff and Papadimitriou determined a tight upper bound, showing that, for all objects whose surfaces are not surfaces of revolution, at most $n_{v}+1$ contacts are necessary [50]. Form closure is impossible to achieve for surfaces of revolution. To emphasize the fact that $n_{v}+1$ contacts are necessary and not sufficient, consider grasping a cube with seven or more points of contact. If all contacts are on one face, then clearly the cube is not form closed.

\subsection{First-Order form Closure Tests}

Because form closure grasps are very secure, it is desirable to design or synthesize such grasps. To do this, one needs a way to test candidate grasps for form closure, and rank them so that the best grasp can be chosen. One reasonable measure of form closure can be derived from the geometric interpretation of the condition (9). The null space constraint and the positivity of $\lambda_{n}$ represent the addition of the columns of $G_{n}$ scaled by the components of $\lambda_{n}$. Any choice of $\lambda_{n}$ closing this loop is in $\mathrm{N}\left(G_{n}\right)$. For a given loop, if the magnitude of the smallest component of $\lambda_{n}$ is positive, then the grasp has form closure, otherwise it does not. Let us denote this smallest component by $\mathrm{d}$. Since such a loop, and hence $\mathrm{d}$, can be scaled arbitrarily, $\lambda_{n}$ should be bounded for computational expediency.

After verifying that $G_{n}$ has full row rank, a quantitative form closure test based on the above observations can be formulated as a linear program (LP) in the

$$
\begin{aligned}
& \text { LP1: maximize : } d \\
& \text { subject to: } G_{n} \lambda_{n}=0 \\
& I \lambda_{n}-1 d \geq 0 \\
& d \geq 0 \\
& 1^{\top} \lambda_{n} \leq n_{c},
\end{aligned}
$$

Where $I \in \mathbb{R}^{n c \times n c}$ is the identity matrix and $I \in \mathbb{R}_{c}^{n}$ a vector with all components equal to 1 . The last inequality is designed to prevent this LP from becoming unbounded. A typical LP solution algorithm determines infeasibility or unboundedness of the constraints in the, so-called, phase I of the algorithm, and considers the result before attempting to calculate an optimal value [51]. If LP1 is infeasible, or if the optimal value $d *$ is zero, then the grasp is not form closed. The quantitative form closure test (10-14) has $n_{c}+8$ constraints and $n_{c}+1$ unknowns. For a typical grasp with $n_{c}<10$, this is a small linear program that can be solved very quickly using the simplex method. However, one should note that the metric d* is dependent on the choice of units used when forming $G_{n}$. It would be advisable to non-dimensionalize the components of the wrenches to avoid dependence of the optimal $\mathrm{d}$ on ones choice of units. This could be done by dividing the first three rows of $\mathrm{G}$ by a characteristic force and the last three rows by a characteristic moment. However, if one desires a binary test, LP1 can be converted into one by dropping the last constraint (14) and applying only phase I of the simplex algorithm. In summary, form closure testing is a two-step process [32]:

\subsection{Form Closure Test}

1. Compute rank $\left(G_{n}\right.$.).

a) If $\operatorname{rank}\left(G_{n}.\right) \neq n_{v}$, then form closure does not exist. Stop.

b) If rank $\left(G_{n}.\right)=n_{v}$, continue.

2. Solve $L P 1$.

a) If $d^{*}=0$, then form closure does not exist.

b) If $d^{*}>0$, then form closure exists and $d^{*}$ is a crude measure of how far the grasp is from losing form closure.

Variations of the Test: If the rank test fails, then the grasp could have partial form closure over as many as rank $\left(G_{n}\right.$.) degrees of freedom. If one desires to test this, then LP1 must be solved using a new $G_{n}$. formed by retaining only the rows corresponding to the degrees of freedom for which partial form closure is to be tested. If $\mathrm{d}^{*}>0$, then partial form closure exists. A second variation is to constrain $d$ to be greater than some large negative value. If this is done, then $\mathrm{d}^{*}<0$ is a crude measure of how far a grasp is from achieving form closure.

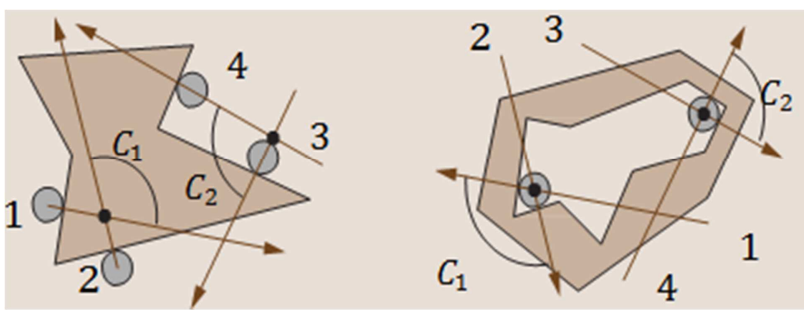

Fig. 5. Planar grasps with first-order form closure. 


\subsection{Planar Simplifications}

In the planar case, Nguyen [52] developed a graphical qualitative test for form closure. Figure 5 shows two form closure grasps with four contacts [53]. To test form closure one partitions the normals into two groups of two. Let $C_{1}$ be the nonnegative span of two normals in one pair and $C_{2}$ be the nonnegative span of the other pair. A grasp has form closure if and only if $C_{1}$ and $C_{2}$ or $-C_{1}$ and $-C_{2}$ see each other for any pairings. Two cones see each other if the open line segment defined by the vertices of the cones lies in the interior of both cones. In the presence of more than four contacts, if any set of four contacts satisfies this condition, then the grasp has form closure. Notice that this graphical test can be difficult to execute for grasps with more than four contacts. Also, it does not extend to grasps of three-dimensional (3D) objects and does not provide a closure measure.

\subsection{Position and Force Control}

In addition to the rich tactile sensations that are described earlier, humans excel at manipulation because they can move competently through free space but quickly transition to regulating grasp force during object contact [53]. Replicating the fluidity of human grasping with a high-impedance parallel jaw gripper $[54,55]$ requires well-designed position and force controllers. Both of these controllers appear several times in the high-level state diagram of Fig. 6; each controller block is labeled with its type, along with the desired motion or force output. To facilitate a generic presentation of our approach, the mathematical constants that are used in this paper are designated with an all-capitalized naming convention [56].

The PR2 gripper $[57,58]$ is a geared mechanism; therefore, it lends itself well to position control. Its position $x_{g}$ in meters and its velocity vg in meters per second was defined. The position is zero when the fingers touch and positive otherwise so that the position value corresponds to the grip aperture. The gripper velocity follows the same sign as position, with positive values indicating that the hand is opening. A good achieve position tracking via a simple proportional-derivative controller with an additional velocity-dependent term to overcome friction

$$
E=K P \cdot\left(x_{g}-x_{g, d e s}\right)+\mathrm{KD} \cdot\left(v_{g}-v_{g, d e s}\right)-\operatorname{sign}\left(v_{g, d e s}\right) \cdot \text { EFRICTION }
$$

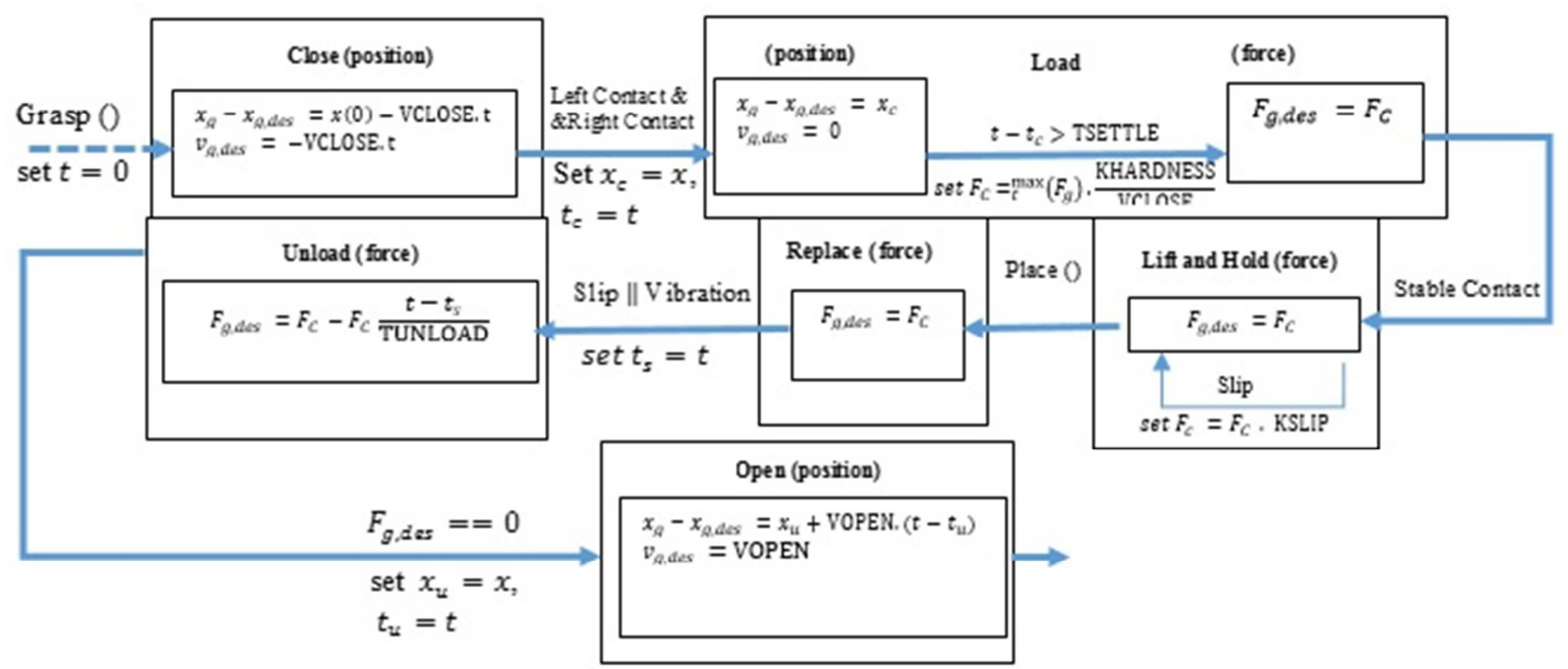

Fig. 6. State diagram of a robotic grasp controller. State transitions occur only after specific tactile events are detected.

Constant-valued parameters, such as VCLOSE, are defined in Table 2.

Here, $E$ is the motor effort (in Newton), KP is the proportional error gain (in Newton's per meter), KD is the derivative error gain (in Newton seconds per meter), and $x_{\text {g,des }}$ and $v_{\text {g,des }}$ are the desired gripper position (in meters) and velocity (in meters per second), respectively. EFRICTION is a scalar constant for feed forward friction compensation, applied to encourage motion in the direction of $v_{\text {g,des }}$. Note that motor effort is defined to be positive in the direction that closes the gripper, which is opposite from the sign convention for the motion variables. Table 2 lists values and units for all of the constants that are used in our controllers, including $\mathrm{KP}, \mathrm{KD}$, and EFRICTION.
Table 2. Valeus chosen for controller constants [59, 60].

\begin{tabular}{llll}
\hline ATHRESH & $4.2 \mathrm{~m} / \mathrm{s}^{2}$ & KHARDNESS & $0.027 \mathrm{~m} / \mathrm{s}$ \\
DLIMIT & $0.02 \mathrm{~N}$ & KP & $20,000 \mathrm{~N} / \mathrm{m}$ \\
EFRICTION & $7.0 \mathrm{~N}$ & KSLIP & 1.08 \\
FBPTHRESH & $0.25 \mathrm{~N}$ & SLIPTHRESH & 0.01 \\
FLIMIT & $0.75 \mathrm{~N}$ & TSETTLE & $0.05 \mathrm{~s}$ \\
FTHRESH & $0.15 \mathrm{~N}$ & TUNLOAD & $0.20 \mathrm{~s}$ \\
KD & $5.000 \mathrm{Ns} / \mathrm{m}$ & VCLOSE & $0.04 \mathrm{~m} / \mathrm{s}$ \\
KFCLOSE & $0.0013 \mathrm{~m} / \mathrm{Ns}$ & VOPEN & $0.05 \mathrm{~m} / \mathrm{s}$ \\
KFOPEN & $0.0008 \mathrm{~m} / \mathrm{Ns}$ & VTHRESH & $0.001 \mathrm{~m} / \mathrm{s}$ \\
\hline
\end{tabular}

A force controller on top of this position controller was created to enable the PR2 to better interact with delicate objects. This controller requires access to the fingertip force signals $F_{g}$ that are described earlier in Section IV-A. Forces 
that compress the fingertips are defined to be positive so that positive motor effort has the tendency to create positive fingertip forces. A force controller that drives the desired position and velocity terms that are based on the error between the desired force and the actual force have been developed [61].

$$
\begin{gathered}
F_{g, \min }=\min \left(F_{g l}, F_{g r}\right) \\
v_{g, \text { des }}=K F \cdot\left(F_{g, \text { min }}-F_{g, \text { des }}\right) \\
\mathrm{KF}=\left\{\begin{array}{c}
\mathrm{KFCLOSE} \text { if } F_{g, \text { min }}-F_{g, \text { des }}<0 \\
\text { KFOPEN otherwise. }
\end{array}\right.
\end{gathered}
$$

We servo on the minimum of the two finger forces $\boldsymbol{F}_{\boldsymbol{g}, \boldsymbol{m i n}}$, as defined in (16), to ensure dual-finger contact. Errors to track the desired force $\boldsymbol{F}_{\boldsymbol{g} \text {,des }}$ are multiplied by the constant gain $\mathrm{KF}$ to yield the desired velocity for the position controller (17). This desired velocity is integrated over time to provide the position controller with a desired grip aperture $\boldsymbol{x}_{\boldsymbol{g} \text {,des }}$. Experimental testing revealed that high values of the gain KF improved force tracking but caused the commonly encountered force-controller effect of chattering as well. It should be noted that an asymmetric gain definition (18), where KFCLOSE is greater than KFOPEN, allows for the best balance of stability and responsiveness during grasping.

\section{Force Closure}

\subsection{Force Closure Definition}

A grasp has force closure, or is force closed, if the grasp can be maintained in the face of any object wrench. Force closure is similar to form closure, but relaxed to allow friction forces to help balance the object wrench. A benefit of including friction in the analysis is the reduction in the number of contact points needed for closure. A three-dimensional object with six degrees of freedom requires seven contacts for form closure, but for force closure, only two contacts are needed if they are modeled as soft fingers [62, 63], and only three (non-collinear) contacts are needed if they are modeled as hard fingers. Force closure relies on the ability of the hand to squeeze arbitrarily tightly in order to compensate for large applied wrenches that can only be resisted by friction. Figure 8 shows a grasped polygon. Consider applying a wrench to the object that is a pure force acting upward along the $y$-axis of the inertial frame. It seems intuitive that, if there is enough friction, the hand will be able to squeeze the object with friction forces preventing the object's upward escape. Also, as the applied force increases in magnitude, the magnitude of the squeezing force will have to increase accordingly.

Since force closure is dependent on the friction models, common models will be introduced before giving formal definitions of force closure.

\subsection{Friction Models}

Recall the components of force and moment transmitted through contact $i$ under the various contact models given earlier $[65,66]$ (Table 3). At contact point $i$, the friction law imposes constraints on the components of the contact force and moment.

Table 3. Vectors of contact force and moment components, also known as the wrench intensity vector, transmitted through contact $i$.

\begin{tabular}{ll}
\hline Model & $\lambda_{i}$ \\
PwoF & $\left(f_{\text {in }}\right)$ \\
$\mathrm{HF}$ & $\left(f_{\text {in }} f_{\text {it }} f_{\text {io }}\right)^{\top}$ \\
$\mathrm{SF}$ & $\left(f_{\text {in }} f_{\text {it }} f_{\text {io }} m_{\text {in }}\right)^{\top}$ \\
\hline
\end{tabular}

Specifically, the frictional components of $\lambda_{i}$ are constrained to lie inside a limit surface, denoted by $L_{i}$, that scales linearly with the product $\mu_{i} f_{i n}$, where $\mu_{i}$ is the coefficient of friction at contact $i$. In the case of Coulomb friction, the limit surface is a circle of radius $\mu_{i} f_{i \mathrm{n}}$. The Coulomb friction cone $F_{i} \mathrm{~s}$ a subset of $\mathrm{R}^{3}$ : [66]

$$
F_{i}=\left\{\left(f_{\text {in }}, f_{i \mathrm{t}}, f_{i \mathrm{o}}\right) \mid \sqrt{f_{i \mathrm{t}}^{2}+f_{i \mathrm{o}}^{2}} \leq \mu_{i} f_{\text {in }}\right\}
$$

More generally, the friction laws of interest have limit surfaces defined in the space of friction components, $\mathbb{R}^{l_{i}-1}$, and friction cones $F_{i}$ defined in the space of $\lambda_{i}, \mathbb{R}^{l_{i}}$. They can be written as: [67]

$$
F_{i}=\left\{\lambda_{i} \in \mathbb{R}^{l i} \mid\left\|\lambda_{i}\right\| w \leq f_{i n}\right\}
$$

where $\left\|\lambda_{i}\right\| \omega$ denotes a weighted quadratic norm of the friction components at contact $i$. The limit surface is defined by $\left\|\lambda_{i}\right\| \omega=$ fin.

Table 4 defines useful weighted quadratic norms for the three contact models: PwoF, HF, and SF. The parameter $\mu_{i}$ is the friction coefficient for the tangential forces, $v_{i}$ is the torsional friction coefficient, and $\mathrm{a}$ is the characteristic length of the object that is used to ensure consistent units in the terms of the norm of the SF model.

\subsection{A Force Closure Definition}

One common definition of force closure can be stated simply by modifying condition (8) to allow each contact force to lie in its friction cone rather than along the contact normal. Because this definition does not consider the hand's ability to control contact forces, this definition will be referred to as frictional form closure. A grasp will be said to have frictional form closure if and only if the following conditions are satisfied: [67]

$$
\left.\begin{array}{rl}
G \lambda & =-g \\
\lambda & \in \mathrm{F}
\end{array}\right\} \forall g \in \mathbb{R}^{n_{v}}
$$

where $\mathrm{F}$ is the composite friction cone defined as: $F=F 1 \times$. $\cdots \times F n_{c}=\left\{\lambda \in R m \mid \lambda_{i} \in F_{i} ; i=1, \ldots, n_{c}\right\}$, and each $F i$ is defined by (20) and one of the models listed in Table 4.

Letting $\operatorname{Int}(F)$ denote the interior of the composite friction cone, Murray et al. give the following equivalent definition [35]: 
Table 4. Norms for the three main contact models.

\begin{tabular}{ll}
\hline Model & $\left\|\lambda_{i}\right\| \omega$ \\
PwoF & 0 \\
HF & $\frac{1}{\mu_{i}} \sqrt{f_{i t}^{2}+f_{i o}^{2}}$ \\
SF & $\frac{1}{\mu_{i}} \sqrt{f_{i t}^{2}+f_{i o}^{2}}+\frac{1}{a v_{i}}\left|m_{i n}\right|$ \\
\hline
\end{tabular}

Friction. Figure 8 shows a grasped polygon. Consider applying a wrench to the object that is a pure force acting upward along the y-axis of the inertial frame. It seems intuitive that, if there is enough friction, the hand will be able to squeeze the object with friction forces preventing the object's upward escape. Also, as the applied force increases in magnitude, the magnitude of the squeezing force [68] will have to increase accordingly.

Since force closure is dependent on the friction models, common models will be introduced before giving formal definitions of force closure.

\subsection{Approximate Force Closure Tests}

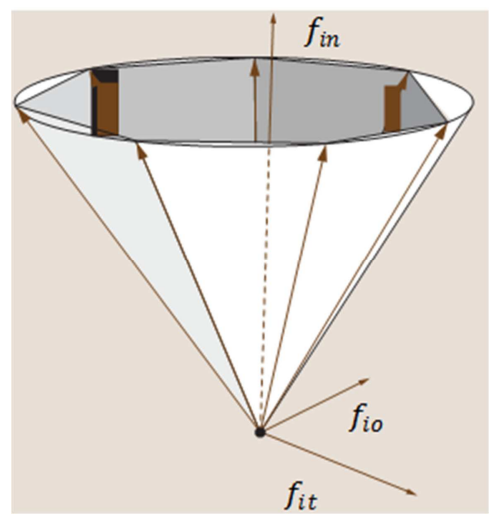

Fig. 7. Quadratic cone approximated as a polyhedral cone with seven generators.

where the index $\mathrm{k}$ varies from 1 to $n_{g}$. If one prefers to approximate the quadratic friction cone by circumscribing polyhedral cone, one simply replaces $\mu_{i}$ in the above definition with $\mu_{i} / \cos \left(\pi n_{g}\right)$. The adjustment needed for the SF model is quite simple. Since the torsional friction in this model is decoupled from the tangential friction, its generators

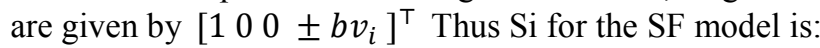

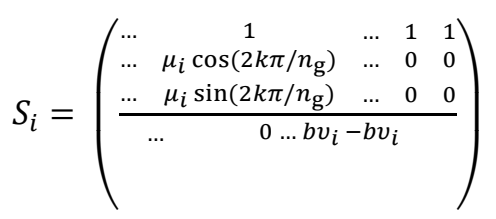

where $b$ is the characteristic length used to unify units. The set of total contact wrenches that may be applied by the hand without violating the contact friction law at any contact can be written as:

Where $S=(S 1, \cdots, S n g)$ and $\sigma=\left(\sigma_{1}^{\top} \ldots \sigma_{n_{\mathrm{g}}}^{\top}\right)^{\top}$. It is convenient to reformulate the friction constraints

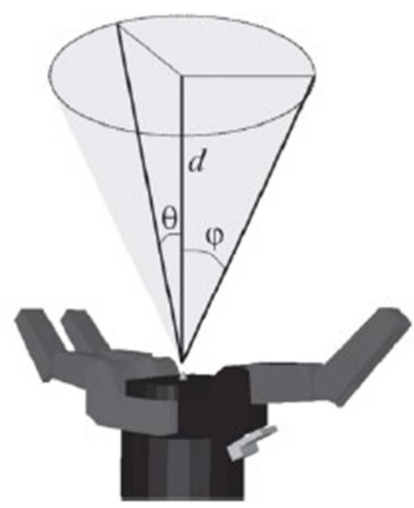

Any of the friction cones discussed can be approximated as the nonnegative span of a finite number $n_{g}$ of generators $s_{i j}$ of the friction cone. Given this, one can represent the set of applicable contact wrenches at contact $i$ as follows:

$$
G_{i} \lambda_{i}=S_{i} \sigma_{i}, \sigma_{i} \geq 0,
$$

where $S i=\left(s_{i 1} \cdots s_{i n_{g}}\right)$ and $\sigma_{i}$ is a vector of nonnegative generator weights. If contact $i$ is frictionless, then $\mathrm{r} n_{g}=1$ and $\left.S_{i}=\left[\hat{n}_{i}^{\top}\left(c_{i}-p\right) \times \hat{n}_{i}\right)^{\top}\right]^{\top}$.

If contact $i$ is of type HF, we represent the friction cone by the nonnegative sum of uniformly spaced contact force generators (Fig.7) whose nonnegative span approximates the Coulomb cone with an inscribed regular polyhedral cone [69]. This leads to the following definition of $S_{i}$ :

$$
S_{i}=\left(\begin{array}{ccc}
\cdots & 1 & \cdots \\
\cdots & \mu_{i} \cos \left(2 k \pi / n_{\mathrm{g}}\right) & \cdots \\
\cdots & \mu_{i} \sin \left(2 k \pi / n_{\mathrm{g}}\right) & \ldots
\end{array}\right)
$$

$$
\boldsymbol{G} \lambda=\boldsymbol{S} \boldsymbol{\sigma}, \boldsymbol{\sigma} \geq \mathbf{0},
$$

In a dual form:

$$
F_{i} \lambda_{i} \geq 0
$$

In this form, each row of $F i$ is normal to a face formed by two adjacent generators of the approximate cone. For an HF contact, row $i$ of $F i$ can be computed as the cross product of $s_{i}$ and $s_{i}+1$. In the case of an SF contact, the generators are of dimension four, so simple cross products will not suffice. However, general methods exist to perform the conversion from the generator form to the face normal form [40].

The face normal constraints for all contacts can be combined into the following compact form: [69]

$$
F \lambda \geq 0 \text {, }
$$

where $\mathrm{F}=$ Blockdiag $\left(F 1, \ldots, F n_{c}\right)$. Let $\boldsymbol{e}_{i} \in \mathbb{R}^{l i}$ be the first row of Hi. Further let $e=\left(e_{1}, \ldots, e_{n c}\right) \in \mathbb{R}^{l}$ and let $\mathrm{E}=$ 
Blockdiag $e=\left(e_{1}, \ldots, e_{n c}\right) \in \mathbb{R}^{l \times n c}$ The following linear program is a quantitative test for frictional form closure. The optimal objective function value $\mathrm{d}^{*}$ is a measure of the distance the contact forces are from the boundaries of their friction cones, and hence a crude measure of how far a grasp is from losing frictional form closure.

LP2: maximize: $\mathrm{d}$

Subject to: $G \lambda=0$

$F \lambda-1 d \geq 0$

$d \geq 0$

$e \lambda \leq n_{c}$.

The last inequality in $L P 2$ is simply the sum of the magnitudes of the normal components of the contact forces. After solving $L P 2$, if $\mathrm{d}^{*}=0$ frictional form closure does not exist, but if $\mathrm{d}^{*}>0$, then it does. If the grasp has frictional form closure, the last step to determine the existence of force closure is to verify the condition $\mathrm{N}(G) \cap \mathrm{N}\left(J^{\mathrm{T}}\right)=0$. If it holds, then the grasp has force closure. This condition is easy to verify with another linear program $L P 3$.

$$
\begin{aligned}
& J^{\top} \lambda=0 \\
& E \lambda-1 d \geq 0 \\
& d \geq 0 \\
& e \lambda \leq n_{c} .
\end{aligned}
$$$$
\text { LP3: maximize: } d
$$$$
\text { Subject to: } G \lambda=0
$$

\subsection{Planar Simplifications [70]}

In planar grasping [71, 72]systems, the approximate method described above is exact. This is because the SF models are meaningless, since rotations about the contact normal would cause motions out of the plane. With regard to the HF model, for planar problems, the quadratic friction cone becomes linear, with its cone represented exactly as:

$$
F_{i}=\frac{1}{\sqrt{1+\mu_{i}^{2}}}\left(\begin{array}{cc}
\mu_{i} & 1 \\
\mu_{i} & -1
\end{array}\right)
$$

Nguyen's graphical form closure test can be applied to planar grasps with two frictional contacts [52]. The only change is that the four contact normals are replaced by the four generators of the two friction cones. However, the test can only determine frictional form closure, since it does not incorporate the additional information needed to determine force closure.

\section{Reviewing the Mathematical Studying for Grasping}

\section{Grasped Polygon in the Plane}

Part 1: G and J

Figure 8 shows a planar hand grasping a polygon [73]. Finger 1 (on the right) contains two joints numbered 1 and 2 . Finger 2 contains joints 3-7, which are numbered in increasing order moving from the palm distally. The inertial frame has been chosen to lie inside the object, with its $\mathrm{x}$-axis passing through contacts 1 and 2, [75] and collinear with the normal vector of contact 2 .

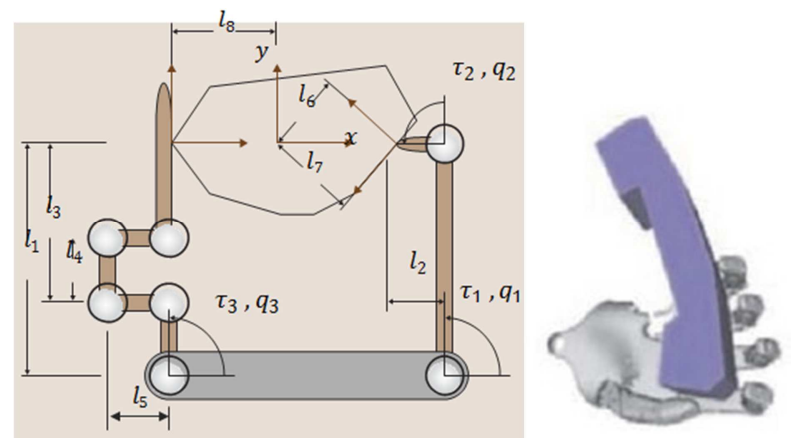

Fig. 8. Planar hand with two fingers and seven joints grasping a polygonal object.

The rotation matrices are given by:

$$
R_{1}=\left(\begin{array}{cc}
-0.8 & -0.6 \\
0.6 & -0.8
\end{array}\right), R_{2}=\left(\begin{array}{ll}
1 & 0 \\
0 & 1
\end{array}\right)
$$

Assuming HF contacts, $G$ is given as

$$
G=\left(\begin{array}{cc|cc}
-0.8 & -0.6 & 1 & 0 \\
0.6 & -0.8 & 0 & 1 \\
l_{6} & -l_{7} & 0 & -l_{8}
\end{array}\right)
$$

It was noted that the first two columns of $\mathrm{G}$ correspond to the normals and tangential unit vectors at contact 1 . The third and fourth columns correspond to contact 2 .

Assuming HF contacts and that all joints are active (i. e., not locked), $\mathrm{J}$ is:

$$
J^{\top}=\left[\begin{array}{cc|cc}
0.8 l_{1} & 0.6 l_{1} & & 0 \\
-0.6 l_{2} & 0.8 l_{2} & & \\
\hline & & -l_{1} & 0 \\
& 0 & -l_{3} & 0 \\
& & -l_{3} & l_{5} \\
& & -l_{3}+l_{4} & l_{5} \\
& -l_{3}+l_{4} & 0
\end{array}\right]
$$

The first two columns of $J^{T}$ are the torques required to produce a unit force in the $\hat{n} 1$ and $\hat{t}_{1}$ directions at contact

1. The horizontal line through the matrix partitions the contributions for the first finger (the upper part) and second finger. Notice that both $J^{\mathrm{T}}$ and $G$ are full column rank.

Part 2: Grasp Classes

This example clearly illustrates the physical qualities of the various grasp classes [74-76] without introducing features that can cloud the descriptions. We now discuss the details of the four grasp classes using the previous planar example. During these discussions it is useful to choose non-dimensional values for the parameters in the grasping system. Assume $l_{4}$ is the unit length. The other lengths as fractions of $l_{4}$ are:

$$
\begin{gathered}
l_{1}=2.7, l_{2}=1.0, l_{3}=1.7, \\
l_{4}=1.0, l_{5}=1.0, l_{6}=1.0, \\
l_{7}=1.3, l_{8}=1.5 .
\end{gathered}
$$

Redundant

Redundancy exists if $N(J)$ is nontrivial. Assuming that both contacts are hard contacts and that all the joints are active, 
rank $(J)=4$, so $N(J)$ is three dimensional. A basis for $N(J)$ was obtained as

$$
N(J) \approx\left(\begin{array}{ccc}
0 & 0 & 0 \\
0 & 0 & 0 \\
-0.50 & -0.24 & -0.10 \\
0.53 & 0.67 & -0.10 \\
0.48 & -0.49 & -0.02 \\
-0.48 & 0.49 & 0.02 \\
-0.02 & 0.01 & 0.98
\end{array}\right)
$$

Since the first two rows are zero, $N(J)$ does not include motions of the first finger (on the right of the palm). To understand this, assume that the object is fixed in the plane. Then the first finger cannot maintain sticking contact at contact 1 unless its joints are also fixed.

The three nonzero columns corresponding to finger 2 show that there are three basis motions of its joints that allow the finger contact to stick to the object contact. For example, the first column shows that, if joint 3 moves roughly as much as joints 4,5 , and 6 , but in the opposite direction as joints 4 and 5 and in the same direction as joint 6 , while joint 7 is more or less fixed, then contact 2 will be maintained.

As can be seen the finger 2 comprises a parallelogram. For the reason of its geometry, one can easily understand that the vector $\left(\begin{array}{llll}0 & 0 & 0 & -11-11\end{array}\right)^{\top}$ is an element of $N(J)$. The velocity interpretation of this vector is the link of the finger which is connected to the palm, and the link touching the object remain fixed in space, while the parallelogram moves as a simple four-bar mechanism. In the same way, joint actions in $N(J)$ do not affect the contact forces, however cause internal hand velocities. It should be noted that, since $N\left(J^{\top}\right)=0$, the complete space of likely generalized velocities and forces at the contacts could be calculated based on above information.

\section{Indeterminate}

With HF contact models, the system exists graspable. But, substituting the HF models with PwoF models eliminates the tangent force components in the $\hat{t}_{1}$ and $\hat{t}_{2}$ directions. This simple and effective replacing eliminates columns 2 and 4 from $G$. Therefore it can be inferred that the structure will be indeterminate. The reduced matrix $\left(G_{(1,3)}\right)$ can be expressed as:

$$
N\left(G_{(1,3)}^{\top}\right) \approx\left(\begin{array}{c}
0 \\
-0.86 \\
0.51
\end{array}\right)
$$

As can be inferred that this basis vector relates to moving the object such that the point coincident with the origin of $\{\mathrm{N}\}$ moves straight downward, while the object rotates counterclockwise. Also, if the analogous force and moment were applied to the object, the frictionless contacts cannot maintain equilibrium $[61,65]$.

Graspable [65, 67]

With two HF contact models in force, $N(G)$ is one dimensional and the system is graspable (rank $(\mathrm{G})=3$ ). The null space basis vector of the grasp matrix is as follows:

$$
N(G) \approx\left(\begin{array}{c}
0.57 \\
\frac{0.42}{0.71} \\
0
\end{array}\right)
$$

The physical interpretation of this basis vector consist of two opposing forces acting through the two contact points. It is worth noting that the contact model is kinematic, and there is not consideration of contact friction. Given that the direction of the contact normal with regard to the line of the internal force, it can be inferred that if the coefficient of friction isn't more than 0.75 , pressing firmly will cause sliding at contact 1 .

Defective [77]

In a defective grasp, $\mathrm{N}\left(\mathrm{J}^{\mathrm{T}}\right) \neq 0$. Given that the original $J$ is full row rank, the grasp is not defective. However, it can be made defective by locking a number of joints and/or changing the hand's configuration so that $J$ is no longer full rank. For example, locking joints $4,5,6$, and 7 makes finger 2 a single-link finger with only joint 3 active. In this new grasping system, $J_{(1,2,3)}^{\top}$ is simply the first three rows of the original $J^{\mathrm{T}}$ given in (28), where the subscript is the list of indices of active joints. The null space basis vector is:

$$
N\left(J_{(1,2,3)}^{\top}\right)=\left(\begin{array}{l}
0 \\
0 \\
0 \\
1
\end{array}\right)
$$

Would be impossible for the hand to give the contact point 2 on the object a velocity in the $\hat{t} 2$-direction while maintaining the contact. This is also clear from the arrangement of joint 3 , contact 2 , and the direction of the contact normal.

The dual interpretation is that forces in $N\left(J^{\top}\right)$ are resisted by the structure and the corresponding joint loads is zero, or equivalently that those forces are not controllable by the hand. Notice that if the model of contact 2 were changed to point without friction, then $N\left(J_{(1,2,3)}^{\top}\right)=0$ and the system would no longer be defective.

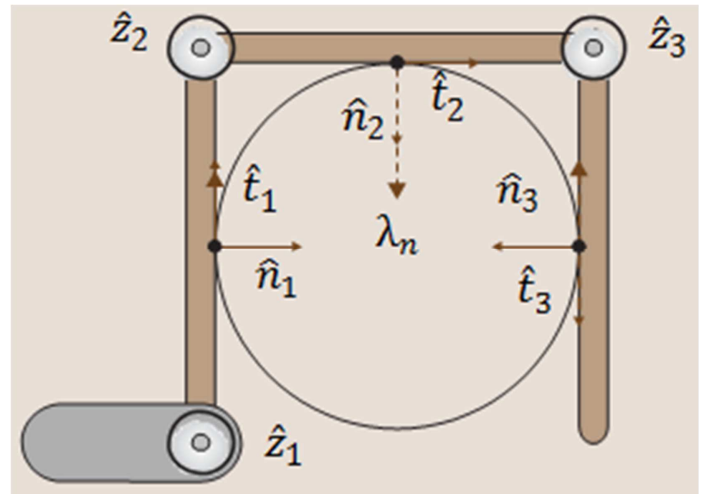

Fig. 9. A sphere grasped by a finger with three revolute joints. The force direction $\lambda_{h}$ (dashed line) is a force that belongs to both $N(G)$ and $N\left(J^{\top}\right)$ and causes hyper staticity.

\section{Conclusion and Future Works}

In this paper, a review for schematics models of different and various types for grasping of varying geometric objects. 
Hence, we analyzed and evaluated models of two-fingered end-effector, three-fingered and multi fingered to autonomously grasps structure and geometrical position of objects. In order to understand the mathematical studying for grasping such as grasped polygon in the plane, and hyper static grasping are investigated in details.

A great deal of understanding of grasping systems can be derived from the simple linear kinematic, dynamic, and contact models [78]. The most commonly used grasp classifications and closure properties can all be derived from the rigid-body assumption. Linearizing these prototypes clues to metrics and tests that can be computed by using linear programming technique and also computational linear algebra.

Contact friction models are not fairly as simple as the Coulomb approximation so widely adopted. For instance, if a contact has to resist a moment approximately its normal, its effective tangential friction coefficient is diminished [78]. It is worth noting that the quadratic Coulomb friction cone was approximated by a polyhedral cone. The analysis problems are hard enough when using the quadratic cone, however they are reasonably amenable [79].

As the future work could be got several objects instantaneous or solving force distribution problem with nonlinear friction cone limitations by taking benefit of second-order cone programming and other related techniques. The other future work can be investigated is grasping in micro-robot. Since today micro-robots cannot grasp all objects.

\section{Appendix}

For more information about for Hyperstatic Grasps see [74-76]:

http://www.tyrazis.com/appendix/appendix.html.

\section{References}

[1] Murray, R.N ., Li, Z., Sastry, S.: A mathematical introduction to robotics manipulation. CRC Press, USA (1994)

[2] Bicchi, A., Kumar, V.: Robotic grasping and contact: a review. In: Robotics and Automation, 2000. Proceedings. ICRA '00. IEEE International Conference on. pp. 348-353. IEEE (2000)

[3] Prattichizzo, D., Trinkle, J.: Springer Handbook of Robotics, pp. 671-700. Springer, Berlin (2008)

[4] Roa Garzón, M.: Grasp planning methodology for 3D arbitrary shaped objects. Ph. d. thesis, Universidad Politécnica de Cataluña (2009)

[5] Haghighi, Alireza Shourangiz, Amin Haghnegahdar, Reza Jahromi Bosheri, and Iman Zare. "Micro-embedded Skimmer in Autonomous Underwater Micro-robots." International Journal of Science and Qualitative Analysis 1, no. 3 (2015): 43-53.

[6] Haghighi, A. Shourangiz, I. Zare, Mohammad Ahmadi Balootaki, Mohammad Orak, and Omid Zare. "Modeling of Bio-inspired Thunnus Albacares and Inchworm-gammarus with Micro Actuators in One Structure." International Journal of Science and Qualitative Analysis 1, no. 3 (2015): 54-63.
[7] Alireza Shourangiz Haghighi, Iman Zare, AliReza Fallahi, Reza Jahromi Bosheri, Amin Haghnegahdar, "Dynamic modeling of flexible tail for bio-inspired dogfish shark (squalus Acanthias)-inchworm with multifunctional locomotion." In Electrical and Electronics Engineering, 2015. ICEEE 2015. 7th Iranian Conference on, pp. 126-132. IEEE, 2015.

[8] AliReza Shourangiz Haghighi, Iman Zare, AliReza Fallahi, Hamid Reza Naji, Amin Bahreini. "Bio-inspired micro-robot with micro-actuators ICPF and floating collector Skimmer." In Electrical and Electronics Engineering, 2015. ICEEE 2015. 7th Iranian Conference on, pp. 133-139. IEEE, 2015.

[9] R. Vertechy, V. Parenti-Castelli, Static and stiffness analyses of a class of over-constrained parallel manipulators with legs of type US and UPS, in: Proceedings of IEEE International Conference on Robotics and Automation (ICRA), 2007, pp. 561-567.

[10] Yoshikawa, T. (2010). Multifingered Robot Hands: Control for Grasping and Manipulation. Annual Reviews in Control, 34(2), 199-208.

[11] Hasegawa, Y., Shikida, M., Shimizu, T., Miyaji, T., Sakai, H., Sato, K., and Itoigawa, K. (2004). A Micromachined Active Tactile Sensor for Hardness Detection, Sensors and Actuators (A Physical), 114(2-3), 141-146.

[12] Nikandrova, Ekaterina, and Ville Kyrki. "Category-based task specific grasping." Robotics and Autonomous Systems 70 (2015): 25-35.

[13] Klingbeil, Ellen, Deepak Rao, Blake Carpenter, Varun Ganapathi, Andrew Y. Ng, and Oussama Khatib. "Grasping with application to an autonomous checkout robot." In Robotics and Automation (ICRA), 2011 IEEE International Conference on, pp. 2837-2844. IEEE, 2011.

[14] Klingbeil, Ellen, Deepak Rao, Blake Carpenter, Varun Ganapathi, Andrew Y. Ng, and Oussama Khatib. "Grasping with application to an autonomous checkout robot." In Robotics and Automation (ICRA), 2011 IEEE International Conference on, pp. 2837-2844. IEEE, 2011.

[15] M. T. Mason and J. K. Salisbury. Manipulator grasping and pushing operations. In Robot Hands and the Mechanics of Manipulation. The MIT Press, Cambridge, MA, 1985.

[16] S. Haidacher and G. Hirzinger, "Estimating finger contact location and object pose from contact measurements in $3 \mathrm{~d}$ grasping," in Proceedings. IEEE International Conference on Robotics and Automation (ICRA), vol. 2. IEEE, 2003, pp. 1805-1810.

[17] Gabiccini, Marco, Antonio Bicchi, Domenico Prattichizzo, and Monica Malvezzi. "On the role of hand synergies in the optimal choice of grasping forces." Autonomous Robots 31, no. 2-3 (2011): 235-252.

[18] Li, Jia-Wei, Hong Liu, and He-Gao Cai. "On computing three-finger force-closure grasps of 2-D and 3-D objects." Robotics and Automation, IEEE Transactions on 19, no. 1 (2003): 155-161.

[19] Shapiro, Amir, Elon Rimon, and Joel W. Burdick. "Passive force closure and its computation in compliant-rigid grasps." In Intelligent Robots and Systems, 2001. Proceedings. 2001 IEEE/RSJ International Conference on, vol. 3, pp. 1769-1775. IEEE, 2001. 
[20] Liu, Yun-Hui, Miu-Ling Lam, and Dan Ding. "A complete and efficient algorithm for searching 3-D form-closure grasps in the discrete domain." Robotics, IEEE Transactions on 20, no. 5 (2004): 805-816.

[21] A. Bicchi and V. Kumar. Robotic grasping and contact: a review. In International Conference on Robotics and Automation (ICRA), 2000.

[22] A. T. Miller, S. Knoop, P. K. Allen, and H. I. Christensen. Automatic grasp planning using shape primitives. In International Conference on Robotics and Automation (ICRA), 2003.

[23] ROS-Matlab Bridge," http://github.com/nmichael/ipc-bridge

[24] Robot Operating System (ROS)," http://www.ros.org

[25] S. Allin, Y. Matsuoka, and R. Klatzky, "Measuring just noticeable differences for haptic force feedback: Implications for rehabilitation," in Proc. IEEE Hapt. Symp., Mar. 2002, pp. 299-302.

[26] T. Takahashi, T. Tsuboi, T. Kishida, Y. Kawanami, S. Shimizu, M. Iribe, T. Fukushima, and M. Fujita, "Adaptive grasping by mulit fingered hand with tactile sensor based on robust force and position control," in Proc. Int. Conf. Robot. Autom., 2008, pp. 264-271.

[27] X. Zhu, H. Ding, Computation of force closure grasps: An iterative algorithm, IEEE Transactions on Robotics, vol.22, no.1, pp.146-162, 2006.

[28] C. Corcoran and R. Platt, "A measurement model for tracking hand object state during dexterous manipulation," in Proceedings. IEEE International Conference on Robotics and Automation (ICRA), 2010, pp. 4302-4308.

[29] Ghanbari, Ahmad, Ramin Solaimani, Arash Rahmani, and Farshid Tabatabaie. "Design and simulating five-finger robot hand to grasp spherical objects." Life Science Journal 10, no. 3 (2013).

[30] M. Yashima, T. Yamawaki, Task oriented accuracy measure for dexterous Manipulation, Proceeding of the IEEE International conference on robotics and biomimetics, Bangkok, Thailand, February, pp.21-26, 2009.

[31] F.Reuleaux: The Kinematics of Machinery (Macmillan, New York 1876), Republished by Dover, New York (2003)

[32] J. ButterfaÃ, M. Grebenstein, H. Liu, and G. Hirzinger, "Dlr-hand ii: Next generation of a dextrous robot hand," in Proceedings. IEEE International Conference on Robotics and Automation (ICRA), 2001, pp. 109-114.

[33] O. Company, S. Krut, F. Pierrot, Modelling and preliminary design issues of a 4-axis parallel machine for heavy parts handling, Journal of Multibody Dynamics 216 (2002) 1-11.

[34] T. Omata, K. Nagata: Rigid body analysis of the indeterminate grasp force in power grasps, IEEE Trans. Robot. Autom. 16(1), 46-54 (2000)

[35] R.M. Murray, Z. Li, S.S. Sastry: A Mathematical Introduction to Robot Manipulation (CRC Press, Boca Raton 2010).

[36] A. Saxena, "Monocular depth perception and robotic grasping of novel objects," Ph.D. dissertation, Stanford University, pp.123-126, 2009.
[37] E. Rimon, J. Burdick: Mobility of bodies in contact i: A 2nd order mobility index for multiple-finger grasps, IEEE Trans. Robot. Autom. 14(5), 696-708 (1998).

[38] Thomas R. Kurfess Ph.D.,Robotics and automotion handbook, pp.406-409, CRC Press LLC, 2005, ISBN 0-8493-1804-1/05

[39] Belter, D.; Kopicki, M.; Zurek, S.; Wyatt, J. "Kinematically optimised predictions of object motion", Intelligent Robots and Systems (IROS 2014), 2014 IEEE/RSJ International Conference on, on page(s): 4422 - 4427

[40] A.J. Goldman, A.W. Tucker: Polyhedral convex cones. In: Linear Inequalities and Related Systems, ed. by H.W. Kuhn, A.W. Tucker (Princeton Univ., York 1956) pp. 19-40.

[41] R. Platt, A. H. Fagg, and R. Grupen, "Learning grasp context distinctions that generalize," in IEEE-RAS International Conference on Humanoid Robots, pp. 504 - 511, Genova, IEEE,2006.

[42] Montana, David J. "The kinematics of contact and grasp." The International Journal of Robotics Research 7, no. 3 (1988): $17-32$.

[43] León, Beatriz, Joaquín L. Sancho-Bru, Néstor J. Jarque-Bou, Antonio Morales, and Máximo A. Roa. "Evaluation of human prehension using grasp quality measures." International Journal of Advanced Robotic Systems 9 (2012).

[44] Yamada, Takayoshi, Toshiya Taki, Manabu Yamada, Yasuyuki Funahashi, and Hidehiko Yamamoto. "Static stability analysis of spatial grasps including contact surface geometry." Advanced Robotics 25, no. 3-4 (2011): 447-472.

[45] De Leone, Renato, Paola Festa, and Emilia Marchitto. "A bus driver scheduling problem: a new mathematical model and a GRASP approximate solution."Journal of Heuristics 17, no. 4 (2011): 441-466.

[46] Rosales, Carlos, Raúl Suárez, Marco Gabiccini, and Antonio Bicchi. "On the synthesis of feasible and prehensile robotic grasps." In Robotics and Automation (ICRA), 2012 IEEE International Conference on, pp. 550-556. IEEE, 2012.

[47] Miller, Andrew T., Steffen Knoop, Henrik Christensen, and Peter K. Allen. "Automatic grasp planning using shape primitives." In Robotics and Automation, 2003. Proceedings. ICRA'03. IEEE International Conference on, vol. 2, pp. 1824-1829. IEEE, 2003.

[48] Ciocarlie, Matei, Kaijen Hsiao, Edward Gil Jones, Sachin Chitta, Radu Bogdan Rusu, and Ioan A. Şucan. "Towards reliable grasping and manipulation in household environments." In Experimental Robotics, pp. 241-252. Springer Berlin Heidelberg, 2014.

[49] Loureiro, Rui CV, and William S. Harwin. "Reach \& grasp therapy: design and control of a 9-DOF robotic neuro-rehabilitation system." In Rehabilitation Robotics, 2007. ICORR 2007. IEEE 10th International Conference on, pp. 757-763. IEEE, 2007.

[50] X. Markenscoff, L. Ni, C.H. Papadimitriou: The geometry of grasping, Int. J. Robot. Res. 9(1), pp.61-74 (1990)

[51] D.G. Luenberger: Linear and Nonlinear Programming, 2nd edn. (Addison-Wesley, Reading 1984) 
[52] L. Han, J.C. Trinkle, Z. Li: Grasp analysis as linear matrix inequality problems, IEEE Trans. Robot. Autom. 16(6), 663$674(2000)$

[53] Bruno Siciliano, Oussama Khatib, Handbooks of Robotics, Springer, pp. 250-252, 2008

[54] R. S. Johansson and J. R. Flanagan, "Coding and use of tactile signals from the fingertips in object manipulation tasks," Nat. Rev. Neurosci., vol. 10, pp. 345-359, May 2009.

[55] [55] Takaki, Takeshi, and Toru Omata. "Grasp force magnifying mechanism for parallel jaw grippers." In Robotics and Automation, 2007 IEEE International Conference on, pp. 199-204. IEEE, 2007.

[56] Nuttall, A. J. G., and AJ Klein Breteler. "Compliance effects in a parallel jaw gripper." Mechanism and machine theory 38, no. 12 (2003): 1509-1522.

[57] Romano, Joseph M., Kaijen Hsiao, Günter Niemeyer, Sachin Chitta, and Katherine J. Kuchenbecker. "Human-inspired robotic grasp control with tactile sensing." Robotics, IEEE Transactions on 27, no. 6 (2011): 1067-1079.

[58] Sanchez, Jose, Sven Schneider, and Paul Plöger. "Safely Grasping with Complex Dexterous Hands by Tactile Feedback." In RoboCup 2014: Robot World Cup XVIII, pp. 332-344. Springer International Publishing, 2015.

[59] Klingbeil, Ellen, Deepak Rao, Blake Carpenter, Varun Ganapathi, Andrew Y. Ng, and Oussama Khatib. "Grasping with application to an autonomous checkout robot." In Robotics and Automation (ICRA), 2011 IEEE International Conference on, pp. 2837-2844. IEEE, 2011.

[60] Miltiadis A. Boboulos, Automation and robotics, pp.83, 2010, ISBN: 978-87-7681-696-4

[61] C. Quennouelle, C. M.Gosselin, Instantaneous Kinemato-Static Model of Planar Compliant Parallel Mechanisms, In: Proceedings of ASME International Design Engineering Technical Conferences, Brooklyn, NY, USA, 2008.

[62] Q. V. Le, D. Kamm, A. Kara, and A. Y. Ng, "Learning to grasp objects with multiple contact points," Robotics and Automation (ICRA), 2010 IEEE International Conference on, pp.5062 - 5069, Anchorage, AK, IEEE, 2010.

[63] Kao, Imin, and Fuqian Yang. "Stiffness and contact mechanics for soft fingers in grasping and manipulation." Robotics and Automation, IEEE Transactions on20, no. 1 (2004): 132-135.

[64] Yoshikawa, Tsuneo, Masanao Koeda, and Hiroshi Fujimoto. "Shape recognition and grasping by robotic hands with soft fingers and omnidirectional camera." InRobotics and Automation, 2008. ICRA 2008. IEEE International Conference on, pp. 299-304. IEEE, 2008.

[65] D. Prattichizzo, A. Bicchi: Dynamic analysis of mobility and grasp ability of general manipulation systems, IEEE Trans. Robot. Autom. 14(2), 241-258 (1998).

[66] A. Bicchi: On the problem of decomposing graspand manipulation forces in multiple whole-limb manipulation, Int. J. Robot. Auton. Syst. 13, 127-147 (1994).
[67] Rosales, C.; Ajoudani, A.; Gabiccini, M.; Bicchi, A. "Active gathering of frictional properties from objects", Intelligent Robots and Systems (IROS 2014), 2014 IEEE/RSJ International Conference on, On page(s): 3982 - 3987.

[68] Brown, Russell G., and Randy C. Brost. "A 3-D modular gripper design tool."Robotics and Automation, IEEE Transactions on 15, no. 1 (1999): 174-186.

[69] Ciocarlie, Matei T., and Peter K. Allen. "Hand posture subspaces for dexterous robotic grasping." The International Journal of Robotics Research 28, no. 7 (2009): 851-867.

[70] S. Thrun, W. Burgard, and D. Fox, Probabilistic Robotics. MIT Press, 2005, Ch. 2. Recursive State Estimation, pp. 13-38.

[71] Sintov, Avishai, Srinivas Raghothama, Roland Menassa, and Amir Shapiro. "A common 3-finger grasp search algorithm for a set of planar objects." InAutomation Science and Engineering (CASE), 2012 IEEE International Conference on, pp. 1095-1100. IEEE, 2012.

[72] Christopoulos, Vassilios N., and Paul Schrater. "Handling shape and contact location uncertainty in grasping two-dimensional planar objects." In Intelligent Robots and Systems, 2007. IROS 2007. IEEE/RSJ International Conference on, pp. 1557-1563. IEEE, 2007.

[73] Phoka, Thanathorn, Pawin Vongmasa, Chaichana Nilwatchararang, Peam Pipattanasomporn, and Attawith Sudsang. "Optimal independent contact regions for two-fingered grasping of polygon." Robotica 30, no. 06 (2012): 879-889.

[74] Zisimatos, A.G.; Liarokapis, M.V.; Mavrogiannis, C.I.; Kyriakopoulos, K.J. "Open-source, affordable, modular, light-weight, underactuated robot hands", Intelligent Robots and Systems (IROS 2014), 2014 IEEE/RSJ International Conference on, On page(s): $3207-3212$

[75] Bard, C.; Troccaz, J.; Vercelli, G. "Shape analysis and hand preshaping for grasping", Intelligent Robots and Systems '91. 'Intelligence for Mechanical Systems, Proceedings IROS '91. IEEE/RSJ International Workshop on, On page(s): 64 - 69 vol.1

[76] Grosso, E.; Vercelli, G. "Grasping strategies for reconstructed unknown 3D objects", Intelligent Robots and Systems '91. 'Intelligence for Mechanical Systems, Proceedings IROS '91. IEEE/RSJ International Workshop on, On page(s): 70 - 75 vol.1

[77] Bruno Siciliano, Oussama Khatib, Handbooks of Robotics, Springer, pp. 297-298, 2008.

[78] G.F. Liu, J. Xu, X. Wang, Z.X. Li: On quality functions for grasp synthesis, fixture planning, and coordinated manipulation, IEEE Trans. Autom. Sci. Eng. 1(2), 146-162 (2004).

[79] L. Han, J.C. Trinkle, Z. Li: Grasp analysis as linear matrix inequality problems, IEEE Trans. Robot. Autom. 16(6), 663674 (2000). 